\title{
Probing Elastic Anisotropy from Defect Dynamics in Langmuir Monolayers
}

\author{
Jan Brugués, ${ }^{1,2}$ Jordi Ignés-Mullol, ${ }^{1,3, *}$ Jaume Casademunt, ${ }^{1,2}$ and Francesc Sagués ${ }^{1,3}$ \\ ${ }^{1}$ Institut de Nanociència i Nanotecnologia de la UB (IN $\left.{ }^{2} U B\right)$, Universitat de Barcelona, 08028 Barcelona, Spain \\ ${ }^{2}$ Departament d'Estructura i Constituents de la Matèria, Universitat de Barcelona, 08028 Barcelona, Spain \\ ${ }^{3}$ Departament de Qímica Física, Universitat de Barcelona, 08028 Barcelona, Spain
}

(Received 23 February 2007; published 22 January 2008)

\begin{abstract}
We study the dynamics of annihilation of point defects in Langmuir monolayers. The absence of hydrodynamic effects allows us to quantitatively relate the asymmetry in defect mobility to the elastic anisotropy of the material, which in turn can be varied through the control of the surface pressure applied to the monolayer. Using the proposed theoretical analysis, we are able to obtain rather elusive equilibrium properties out of relatively simple dynamical measurements. In particular, we measure the elastic constants and their pressure dependence.
\end{abstract}

PACS numbers: 61.30.Jf, 61.72.Cc, 68.18.-g, 68.35.Md

The study of defect dynamics is of general relevance in many areas of physics. Of special interest is the study of the interaction and annihilation of defect pairs. A remarkable feature of this process, both observed experimentally and numerically, although not fully understood, is the enhanced mobility of the positively charged defect with respect to its negative counterpart. In general, elastic anisotropy (inequality of the elastic constants) and hydrodynamic effects arising from defect motion (backflow) play a role in explaining this asymmetry. In fact, the latter is dominant in the context of bulk liquid crystals [1-4], thus hindering the possibility to develop a simple method to quantitatively relate material elasticity to defect dynamics, which would be an interesting alternative to traditional methods of determining the elastic constants $[5,6]$. We address here the somewhat opposite scenario by studying defect dynamics in Langmuir monolayers spread at the air-water interface [7]. Contrary to bulk liquid crystals, where rotational and translational viscosities are of the same order of magnitude, local molecular rotation without hydrodynamic effects is possible in Langmuir monolayers and, thus, defect dynamics can be simply traced back to the effect of material elasticity. As a result, measurements of differential defect mobilities enable us to probe the elastic anisotropy of the two-dimensional material.

Here, we report on quantitative studies of defect dynamics in a particular class of Langmuir monolayers with a polar nematic arrangement. The pronounced observed asymmetry of defect mobility is rationalized in terms of a simple theoretical model that rules out backflow effects. Indeed, coupling with the aqueous subphase hinders any monolayer flow arising from in-plane rotation that accompanies defect motion [8]. The model is exploited to extract the dependence of the elastic anisotropy on surface pressure and to estimate the compressibility-dependent elastic constants of the compressible 2D system. This indirect procedure is demonstrated to be an advantageous alternative over direct measurements, unpractical in these quasitwo-dimensional systems.
Experiments are performed in a shallow thermostated Teflon cuvette where the monolayer is spread over an area delimited by two mobile barriers, which control the surface pressure. The monolayer is a cis and trans mixture of the photosensitive azobenzene amphiphile $8 \mathrm{Az} 3 \mathrm{COOH}$ [9]. Monolayers feature isolated birefringent trans-rich domains (henceforth droplets) surrounded by an isotropic cis-rich medium [10]. Mesoscale organization inside the droplets is probed with a custom-built Brewster angle microscope (BAM) [11].

For a wide range of experimental conditions, the organization of the amphiphilic molecules inside the circular domains is characterized by a uniform tilt (around $45^{\circ}$ [9]) with respect to the air-water interface normal. Molecular ordering can thus be described by a two-dimensional vector field. Constant-angle anchoring at the droplet boundary $[9,12]$ results in the inclusion of inner point defects of total charge $+1[13,14]$. Small enough droplets feature a single $s=+1$ defect near the center, and a pure bend texture around the core (see Fig. 1).

Droplets with opposed chirality fuse abruptly (Fig. 1), creating a pair of new point defects upon intersection [15]. Since the total charge must be preserved inside the closed domain, we consistently assign a $-1 / 2$ charge to each boundary defect. These semi-integer defects are necessarily confined to the droplet boundary since they are not allowed inside polar nematic domains. In the simplest route to collapse, typical in the fusion of droplets of different size [16], one of the originally central $s=+1$ defects is annihilated in a two-step mechanism [Fig. 1(A)]. First it migrates to merge at the boundary with one of the newly created singularities, resulting in a $+1 / 2$ boundary defect. The latter, and the remaining $-1 / 2$ boundary defect approach following an asymmetric dynamics along the contour of the fused droplet, with $+1 / 2$ defects always moving faster [see Fig. 1(B) for a representation of defect trajectories]. Relaxation of the droplet shape following coalescence is much faster than defect dynamics. As a result, droplet circularity does not change measurably dur- 

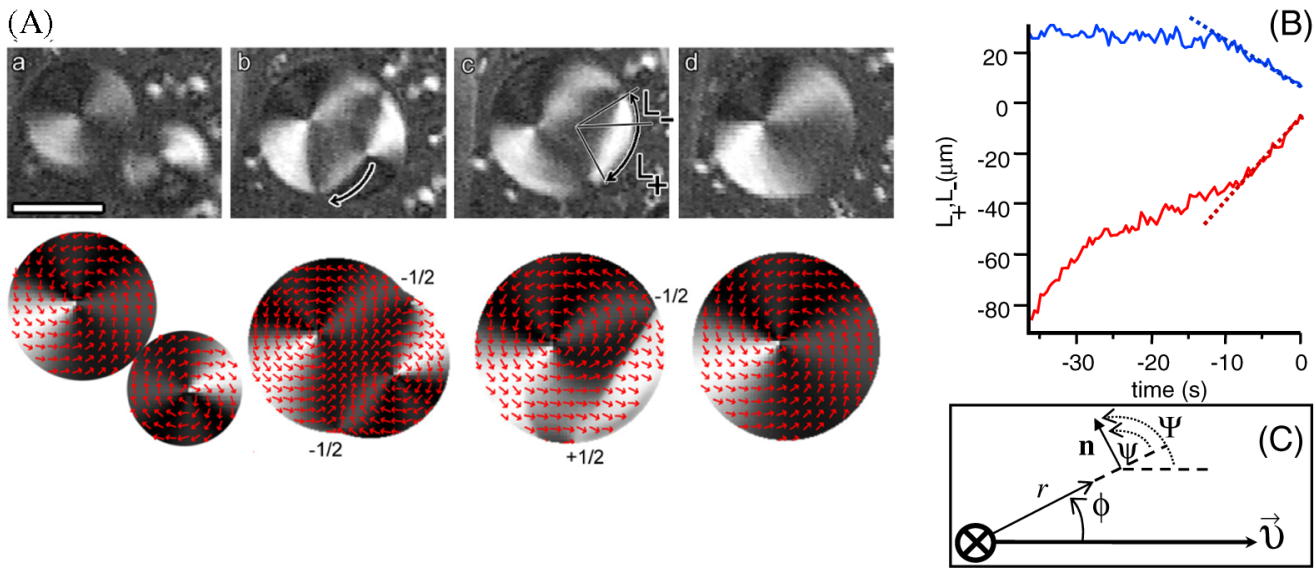

FIG. 1 (color online). (A) Experimental coalescence process of a clockwise and an anticlockwise bend domain at $T=32{ }^{\circ} \mathrm{C}$ and $\Pi=0.3 \mathrm{mN} \mathrm{m}^{-1}$ leading to a droplet with a single $s=+1$ defect [28]. BAM analyzer is set at $60^{\circ}$ counterclockwise from the plane of incidence, which includes the vertical axis in the images. A sketch of the molecular field is shown below each experimental image. Merging of one of the inner $s=+1$ defects and one of the two $-1 / 2$ boundary defects created upon fusion (b) results in a $\pm 1 / 2$ pair of boundary defects that attract and annihilate (d) following the arc lengths towards their meeting point shown in (B). The straight lines yield the average linear velocity in the pseudoconstant velocity regime prior to collapse. Elapsed times from panel (b) are $90 \mathrm{~s}(\mathrm{c})$ and $124.6 \mathrm{~s}$ (d). The ruler is $100 \mu \mathrm{m}$ long. Video image digitization and processing [29] is used to set the quasicircular droplet contour as fixed in space. (C) Schematics of the angular coordinates defined in the text.

ing the final stages of defect collapse, where a roughly constant linear velocity along the curved boundary is observed. The ratio of linear velocities in this regime is used below to quantify the asymmetry in defect mobilities.

Recent numerical analysis [2] has shown that the effect of elastic anisotropy and backflow cannot be decoupled in the study of asymmetric defect mobilities in bulk liquid crystals. We will argue that backflow can be neglected in our system and thus one can propose a simplified model where the study of defect dynamics can be directly related to the material elastic constants. Absence of backflow can be justified by comparing the rotational viscosity in our system $\left(\sim 10^{-10} \mathrm{~kg} \mathrm{~s}^{-1}\right.$ [17] $)$ with the much larger effective translational viscosity resulting from the coupling between the monolayer and the subphase [8]. This may be estimated as the subphase viscosity $\left(\sim 10^{-3} \mathrm{~kg} \mathrm{~m}^{-1} \mathrm{~s}^{-1}\right)$ times a characteristic length in the monolayer, i.e., the droplet radius $(\sim 50 \mu \mathrm{m})$. Actually, it has recently been shown that complex reorientations of the molecular field can take place in this system without noticeable hydrodynamic effects [18].

Under the previous assumptions, we propose a generic free energy model that accounts for the lowest order distortions of the director field $\mathbf{n}$,

$$
F=\int d^{2} x\left[\frac{K_{S}}{2}(\nabla \cdot \mathbf{n})^{2}+\frac{K_{B}}{2}(\nabla \times \mathbf{n})^{2}\right],
$$

where $K_{S}$ and $K_{B}$ are the splay and bend constants [5,6]. Earlier studies on this system suggest that the effect of anisotropic boundary conditions can be neglected with respect to inner elasticity in the regimes where pure bend textures are obtained [10]. Strictly speaking, Eq. (1) should include contributions from a density order parameter so as to account for the finite compressibility of this system $[14,19]$. Nevertheless, at a fixed lateral pressure, explicit density contributions can be renormalized into effective elastic constants, and Eq. (1) is of general applicability.

We will employ this free energy in order to propose a model for the differential mobility of boundary defects described above. Figure 1(a) shows that the director field around $\pm 1 / 2$ defects rotates when defects move along the curved boundary. Thus, the director field around a defect moving along the boundary can be mapped into that of a defect in rectilinear motion. On the other hand, each boundary defect can be regarded as a \pm 1 , with half its spatial extend being virtual. As argued below, this allows to extrapolate the results of rectilinear \pm 1 defect motion to that of semi-integer boundary defects.

Let us consider an isolated point defect, and express the director field around it in polar coordinates $(r, \phi)$. Minimization of Eq. (1) gives,

$$
(1+\alpha \cos 2 \psi)\left(\frac{\partial^{2} \psi}{\partial \phi^{2}}\right)=\alpha \sin 2 \psi\left[\left(\frac{\partial \psi}{\partial \phi}\right)^{2}-1\right]
$$

where $\psi$ is the angle between $\mathbf{n}$ and the radial direction [Fig. 1(C)]. Here, $\alpha=\left(K_{S}-K_{B}\right) /\left(K_{S}+K_{B}\right)$ is a measure of the elastic anisotropy. $\alpha<0$ (resp. $>0$ ) favors splay (bend) textures, and $\alpha=0$ is the isotropic case. For $s=$ +1 , the energetically favorable solution to Eq. (2) for $\alpha>$ 0 is $\psi_{+}= \pm \pi / 2$ (pure bend configuration), and $\psi_{+}=0$, $\pi$ for $\alpha<0$ (pure splay). For $s=-1$, the director field around the defect is given by [20] 


$$
\phi\left[\psi_{-}, k_{-}\right] \equiv k_{-} \int_{0}^{\psi_{-}}\left[\frac{1+\alpha \cos 2 x}{1+\alpha k_{-}^{2} \cos 2 x}\right]^{1 / 2} d x,
$$

where the integration constant $k_{-}$is determined by $\phi\left[-4 \pi, k_{-}\right]=2 \pi$. Within a quasistatic approximation, we assume that Eq. (3) describes the instantaneous director field during rectilinear defect motion. The dissipation rate per unit length will be $\Sigma=\gamma \int d S(\partial \Psi / \partial t)^{2}$ [21], where $\Psi$ is the angle between the director field and the polar axis, $\Psi=\psi+\phi$, and $\gamma$ is a rotational viscosity of the medium. Because of the symmetry of Eq. (3), differences in dissipation for $s= \pm 1$ will be encoded in the angular part of $\Sigma$, which is independent of the direction of motion of the defect [22], and reads $\frac{1}{2} \int_{0}^{2 \pi} d \phi(\partial \Psi / \partial \phi)^{2}$. Recalling the definition of the defect charge, $\Psi(\phi+2 \pi)=\Psi(\phi)+$ $2 \pi s$, the fact that $\partial \Psi_{+} / \partial \phi=+1$, and using a general inequality of functional analysis, one finds

$$
\begin{aligned}
\int_{0}^{2 \pi} d \phi\left(\frac{\partial \Psi_{+}}{\partial \phi}\right)^{2} & =\frac{1}{2 \pi}\left[\int_{0}^{2 \pi} d \phi \frac{\partial \Psi_{ \pm}}{\partial \phi}\right]^{2} \\
& \leq \int_{0}^{2 \pi} d \phi\left(\frac{\partial \Psi_{-}}{\partial \phi}\right)^{2}
\end{aligned}
$$

which proves that dissipation will generically be larger for negative defects (with the equality holding for the isotropic case). This results from the fact that anisotropy introduces an inhomogeneous distortion only to the negative defect. Since the dissipation is quadratic on the variation of the director field, and the overall rotation of the director field is fixed by topology, anisotropy increases the dissipation for the negative defect but not for the positive one. This implies that, due to elastic anisotropy, isolated positive defects are always faster than negative ones. The power supplied per unit length needed to maintain the defect moving at a fixed velocity $v$ is $P=f v$, where $f$ is the drag force, and must be equal to the dissipation rate $\Sigma$. This leads to the expressions

$$
\begin{aligned}
& f_{-} \simeq \frac{1}{2} \gamma v_{-} \log \left(\frac{R}{r_{c}}\right) \int_{0}^{2 \pi} d \phi\left[1+\frac{1}{k_{-}} \sqrt{\frac{1+\alpha k_{-}^{2} \cos 2 \psi_{-}}{1+\alpha \cos 2 \psi_{-}}}\right]^{2}, \\
& f_{+} \simeq \pi \gamma v_{+} \log \left(\frac{R}{r_{c}}\right) .
\end{aligned}
$$

Here, $r_{c}$ is the core radius of the defect, and $R$ represents a macroscopic scale, which is taken here as the droplet radius. Regardless of the accuracy of the logarithmic dependence in the drag force expression [23], what is relevant for our analysis of the relative velocities (see below) is the fact that $R$ and $r_{c}$ can be assumed to be roughly the same for the two defects of opposite charges. More accurate estimations of those parameters would result in a residual sublogarithmic dependence on the elastic anisotropy, which can be neglected.

Balance between the drag force and the elastic attraction between defects allows us to express the ratio of the defect velocities in terms of $\alpha$ alone using Eq. (5). Since dissipation results from distortions of the director field, $\pm 1 / 2$

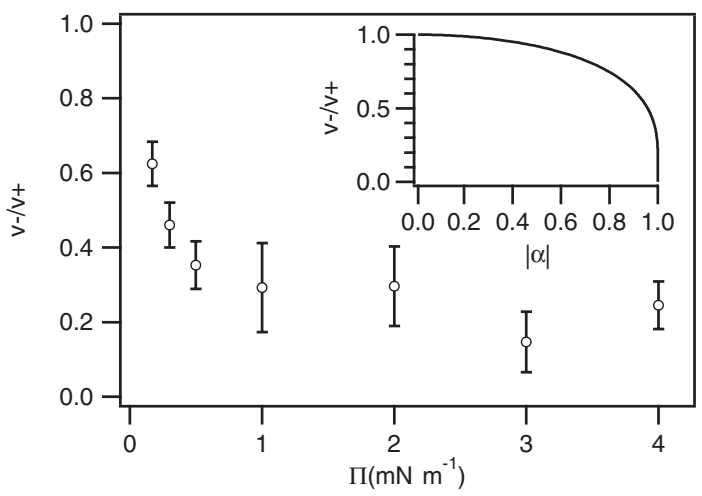

FIG. 2. Experimental measurements of the relative velocities of boundary defects moving towards their annihilation as a function of surface pressure. The inset is the graphical solution of Eq. (6).

boundary defects dissipate half as much as \pm 1 defects so, in both cases,

$$
\frac{v_{+}}{v_{-}}=\frac{1}{2 \pi} \int_{0}^{2 \pi} d \phi\left[1+\frac{1}{k_{-}} \sqrt{\frac{1+\alpha k_{-}^{2} \cos 2 \psi_{-}}{1+\alpha \cos 2 \psi_{-}}}\right]^{2} .
$$

As seen in the inset of Fig. 2, asymmetry is only significant for large $\alpha$. Interestingly, the ratio $v_{+} / v_{-}$is invariant if $K_{B}$ and $K_{S}$ are interchanged [2], i.e., if $\alpha$ is replaced by $-\alpha$ [24].

Although Eq. (6) is of more general validity, its usefulness is limited to regimes of constant defect motion. This is precisely the case in the experiments reported in Fig. 1, so Eq. (6) can now be exploited to determine the ratio $K_{S} / K_{B}$ by measuring defect velocities under different monolayer conditions. To this end we have conducted experiments varying the surface pressure, which is kept below $4 \mathrm{mN} \mathrm{m}^{-1}$. Above this value, the range of motion of the slow defect cannot be resolved in our system. Similarly, temperature is maintained at $32{ }^{\circ} \mathrm{C}$, a compromise between the hindered mobility at lower temperatures and the fast dynamics above this value. Droplet radii were considered in the range 30-60 $\mu \mathrm{m}$, with no significant effect.

Experimental ratios of defect velocities (defined as the linear velocity along the curved boundary) as a function of lateral pressure are shown in Fig. 2 in terms of the mean and standard deviation of several experiments. The ratio $v_{-} / v_{+}$can be transformed, using Eq. (6), to yield $\alpha$ vs $\Pi$ and, therefore, the ratio of the elastic constants as a function of $\Pi$ (Fig. 3). Our measurements can be combined with the results of Feder et al. [17] whose analysis of orientation fluctuations in this system yielded a value for the geometric mean of $K_{S}$ and $K_{B}$ of $(40 \pm 25) k_{B} T$. This way, we can estimate $K_{S}$ and $K_{B}$ as a function of $\Pi$ (Fig. 3). In the zero- $\Pi$ limit (lowest anisotropy) $K_{S} \simeq$ $K_{B} \simeq 10^{-19} \mathrm{~J}$. As $\Pi$ increases, there is a rapid increase (resp. decrease) of $K_{S}$ (resp. $K_{B}$ ) until $\Pi \simeq 1 \mathrm{mN} / \mathrm{m}$, where further variation of these parameters cannot be resolved. These values are consistent with estimations 


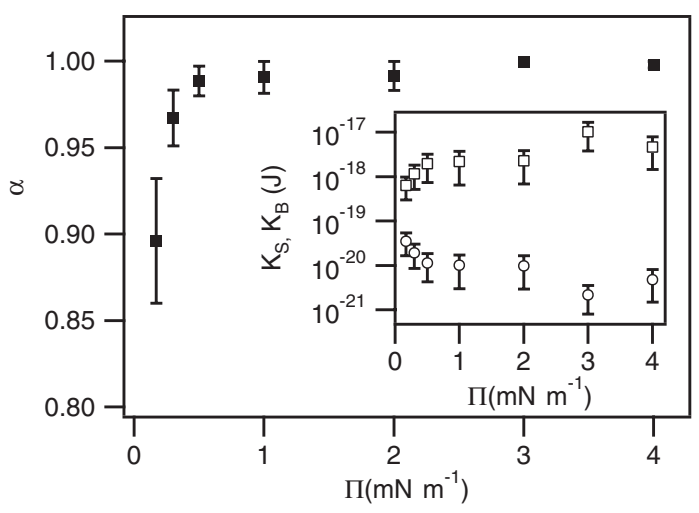

FIG. 3. Anisotropy parameter, $\alpha$, as a function of $\Pi$, as obtained from the data in Fig. 2. The inset shows the value of the elastic constants $K_{S}(\square)$, and $K_{B}(\bigcirc)$ estimated as described in the text.

found in the literature [14] and values extrapolated for thin suspended liquid crystal films [25].

We can qualitatively relate these results to the known tendency of azobenzene derivatives to form supramolecular aggregates ( $H$-aggregates), whose presence can be revealed here by spectroscopy methods [26]. In short, aggregates form in such a way that the azobenzene planes of neighboring molecules are parallel, stacking perpendicularly to the molecular tilting direction. Because of this, a splay arrangement of the molecular field inside the axisymmetric droplets would be energetically more demanding than its bend counterpart, since the former would impose a certain curvature on the $H$ aggregates. The known increase of the extension of aggregates with $\Pi[10]$ can therefore justify the observation that splay distortions are increasingly less favorable. In fact, molecules cease to behave as individual monomers at $\Pi \simeq 2 \mathrm{mN} / \mathrm{m}$, which may justify the levelling off of the elastic constants at moderate pressures. Nevertheless, this qualitative argument cannot explain why the behavior is so dramatically different even with modest extension of the aggregation. Molecular dynamics simulations could be employed to address these issues [27].

In summary, we have studied Langmuir monolayers where defect dynamics can be analyzed in the absence of backflow. As a consequence, differential defect mobilities can be traced back to elastic anisotropy. We have shown that this simplified scenario can be exploited with the aid of a simple model to use dynamical measurements to gain quantitative knowledge of the dependence of rather elusive material parameters, here the elastic constants, on a basic thermodynamic control parameter of the monolayer such as the surface pressure.

We thank M. A. Vallvé for assistance in the experiments. Partial financial support for this research was provided by MEC under Project No. FIS2006-03525 and by DURSI through Projects No. 2005-SGR/00507 (J. B. and J. C.) and
No. 2005-SGR/00653 (J. I-M. and F. S.). J. B. acknowledges support from the Ministerio de Educación y Cultura and J.I-M. acknowledges support from the Ramón y Cajal Program (MEC).

*jignes@ub.edu

[1] G. Toth et al., Phys. Rev. Lett. 88, 105504 (2002).

[2] D. Svensek and S. Zǔmer, Phys. Rev. E 66, 021712 (2002).

[3] P. Oswald and J. Ignés-Mullol, Phys. Rev. Lett. 95, 027801 (2005).

[4] C. Blanc et al., Phys. Rev. Lett. 95, 097802 (2005).

[5] P.G. de Gennes and J. Prost, The Physics of Liquid Crystals (Oxford University, New York, 1995), 2nd ed.

[6] P. Oswald and P. Pieranski, Nematic and Cholesteric Liquid Crystals: Concepts and Physical Properties Illustrated by Experiments (Taylor \& Francis, London, 2005).

[7] E. Hatta and T. M. Fischer, J. Phys. Chem. B 107, 6406 (2003).

[8] H. A. Stone, Phys. Fluids 7, 2931 (1995).

[9] J. Crusats et al., Langmuir 20, 8668 (2004).

[10] J. Ignés-Mullol et al., Langmuir 21, 2948 (2005).

[11] J. Ignés-Mullol et al., J. Phys. Chem. B 108, 612 (2004).

[12] R. Reigada et al., J. Chem. Phys. 121, 9066 (2004).

[13] N. D. Mermin, Rev. Mod. Phys. 51, 591 (1979).

[14] Y. Tabe et al., Phys. Rev. Lett. 82, 759 (1999).

[15] Coalescence of droplets with the same chirality is hindered and seldom observed.

[16] Other scenarios, less amenable to quantitative analysis, have been observed, and will be described elsewhere.

[17] A. Feder et al., Phys. Rev. Lett. 79, 1682 (1997).

[18] P. Burriel et al., Langmuir 22, 187 (2006).

[19] G. A. Hinshaw, Jr., R. G. Petschek, and R. A. Pelcovits, Phys. Rev. Lett. 60, 1864 (1988).

[20] L. D. Landau and E. M. Lifshitz, Theory of Elasticity (Pergamon, New York, 1986), 3rd ed.

[21] M. Kleman and O. D. Lavrentovich, Soft Matter Physics: An Introduction (Springer, New York, 2003).

[22] We have used the symmetry $\psi_{-}[\phi+\pi / 2]=\psi_{-}(\phi)+$ $\pi$.

[23] G. Ryskin and M. Kremenetsky, Phys. Rev. Lett. 67, 1574 (1991).

[24] The change $\alpha \rightarrow-\alpha$ results only in a rotation of $\pi / 2$ of the solution to Eq. (2). Thus the overall rotation of $\mathbf{n}$ due to defect motion is the same, leaving invariant the integrand in Eq. (6).

[25] C. Rosenblatt et al., Phys. Rev. Lett. 42, 1220 (1979).

[26] J. M. Pedrosa et al., J. Phys. Chem. B 106, 2583 (2002).

[27] M. P. Allen et al., J. Chem. Phys. 105, 2850 (1996).

[28] See EPAPS document No. E-PRLTAO-100-045803 for a movie file corresponding to Brewster angle microscopy images shown in Fig. 1 of the manuscript. For more information on EPAPS, see http://www.aip.org/pubservs/ epaps.html.

[29] W.S. Rasband, ImageJ, U.S. N.I.H, Bethesda, Maryland, http://rsb.info.nih.gov/ij/ (1997-2007). 